\title{
Higher topological complexity of hyperbolic groups
}

\author{
Sam Hughes ${ }^{1} \cdot$ Kevin $\mathrm{Li}^{2}$
}

Received: 24 October 2021 / Revised: 11 January 2022 / Accepted: 14 January 2022 /

Published online: 4 February 2022

(c) The Author(s) 2022

\begin{abstract}
We prove for non-elementary torsion-free hyperbolic groups $\Gamma$ and all $r \geq 2$ that the higher topological complexity $\mathrm{TC}_{r}(\Gamma)$ is equal to $r \cdot \operatorname{cd}(\Gamma)$. In particular, hyperbolic groups satisfy the rationality conjecture on the TC-generating function, giving an affirmative answer to a question of Farber and Oprea. More generally, we show that the same conclusions hold for certain toral relatively hyperbolic groups.
\end{abstract}

Keywords Higher topological complexity · Hyperbolic groups · Classifying spaces for families of subgroups

Mathematics Subject Classification 55M30 - 55R35 - 20F67

\section{Introduction}

Let $r \geq 2$ be an integer. The higher (or sequential) topological complexity $\mathrm{TC}_{r}(X)$ of a path-connected space $X$ was introduced by Rudyak (2010), generalising Farber's topological complexity (Farber 2003). The motivation for these numerical invariants arises from robotics. They provide a measure of complexity for the motion planning problem in the configuration space $X$ with prescribed initial and final states, as well as $r-2$ consecutive intermediate states. More precisely, consider the path-fibration $p: X^{[0,1]} \rightarrow X^{r}$ that maps a path $\omega:[0,1] \rightarrow X$ to the tuple $\left(\omega(0), \omega\left(\frac{1}{r-1}\right), \ldots, \omega\left(\frac{r-2}{r-1}\right), \omega(1)\right)$. Then $\mathrm{TC}_{r}(X)$ is defined as the minimal integer $n$ for which $X^{r}$ can be covered by $n+1$ many open subsets $U_{0}, \ldots, U_{n}$ such that $p$

$\bowtie \quad$ Kevin Li

kevin.li@soton.ac.uk

Sam Hughes

sam.hughes@maths.ox.ac.uk

1 Mathematical Institute, University of Oxford, Andrew Wiles Building, Oxford OX2 6GG, UK

2 School of Mathematical Sciences, University of Southampton, Southampton SO17 1BJ, UK 
admits a local section over each $U_{i}$. If no such $n$ exists, one sets $\operatorname{TC}_{r}(X):=\infty$. Note that $\mathrm{TC}_{2}(X)$ recovers the usual topological complexity.

Since the higher topological complexities are homotopy invariants, one obtains interesting invariants of groups $\Gamma$ by setting $\mathrm{TC}_{r}(\Gamma):=\mathrm{TC}_{r}(K(\Gamma, 1))$, where $K(\Gamma, 1)$ is an Eilenberg-MacLane space. The topological complexities $\mathrm{TC}_{r}(\Gamma)$ have been computed for several classes of groups (see e.g. Farber et al. 2019; Farber and Mescher 2020; Dranishnikov 2020 for $r=2$, Farber and Oprea 2019; Aguilar-Guzmán et al. 2021; González et al. 2016 for $r \geq 2$, and references therein). In a celebrated result of Dranishnikov (2020) (see also Farber and Mescher 2020), the topological complexity $\mathrm{TC}_{2}(\Gamma)$ of groups with cyclic centralisers, such as hyperbolic groups, was shown to equal $\operatorname{cd}(\Gamma \times \Gamma)$. Here cd denotes the cohomological dimension. We generalise this result to all higher topological complexities $\mathrm{TC}_{r}(\Gamma)$ for $r \geq 2$, as well as to a larger class of groups containing certain toral relatively hyperbolic groups. Recall that a collection $\left\{P_{i} \mid i \in I\right\}$ of subgroups of $\Gamma$ is called malnormal, if for all $g \in \Gamma$ and $i, j \in I$ we have $g P_{i} g^{-1} \cap P_{j}=\{e\}$, unless $i=j$ and $g \in P_{i}$.

Theorem 1.1 Let $r \geq 2$ and let $\Gamma$ be a torsion-free group with $\operatorname{cd}(\Gamma) \geq 2$. Suppose that $\Gamma$ admits a malnormal collection of abelian subgroups $\left\{P_{i} \mid i \in I\right\}$ satisfying $\operatorname{cd}\left(P_{i}^{r}\right)<\operatorname{cd}\left(\Gamma^{r}\right)$ such that the centraliser $C_{\Gamma}(g)$ is cyclic for every $g \in \Gamma$ that is not conjugate into any of the $P_{i}$. Then $\mathrm{TC}_{r}(\Gamma)=\operatorname{cd}\left(\Gamma^{r}\right)$.

The preceding theorem was obtained for the case $r=2$ by the second author in $\mathrm{Li}$ (2021).

For a space $X$, the TC-generating function $f_{X}(t)$ is defined as the formal power series

$$
f_{X}(t):=\sum_{r=1}^{\infty} \mathrm{TC}_{r+1}(X) \cdot t^{r}
$$

The TC-generating function of a group $\Gamma$ is set to be $f_{\Gamma}(t):=f_{K(\Gamma, 1)}(t)$. Recall that a group $\Gamma$ is said to be of type $F$ (or geometrically finite) if it admits a finite model for $K(\Gamma, 1)$.

Following Farber and Oprea (2019), we say that a finite CW-complex $X$ (resp. a group $\Gamma$ of type $F$ ) satisfies the rationality conjecture if the TC-generating function $f_{X}(t)$ (resp. $\left.f_{\Gamma}(t)\right)$ is a rational function of the form $\frac{P(t)}{(1-t)^{2}}$, where $P(t)$ is an integer polynomial with $P(1)=\operatorname{cat}(X)$ (resp. $P(1)=\operatorname{cd}(\Gamma))$. Here cat denotes the Lusternik-Schnirelmann category. While a counter-example to the rationality conjecture for finite CW-complexes was found in Farber et al. (2020), the rationality conjecture for groups of type $F$ remains open. It is known to hold, e.g. for abelian groups of type $F$, right-angled Artin groups, fundamental groups of closed orientable surfaces, and Higman's group (see Farber and Oprea 2019, Section 8). Our result extends the class of groups for which the rationality conjecture holds as follows.

Corollary 1.2 Let $\Gamma$ be a group as in Theorem 1.1. If $\Gamma$ is of type $F$, then

$$
f_{\Gamma}(t)=\operatorname{cd}(\Gamma) \frac{(2-t) t}{(1-t)^{2}} .
$$


In particular, the rationality conjecture holds for $\Gamma$.

As remarked by Farber and Oprea in (2019, p. 159), it is particularly interesting to determine the validity of the rationality conjecture for the class of hyperbolic groups. We answer their question in the affirmative.

Corollary 1.3 The rationality conjecture holds for torsion-free hyperbolic groups.

\section{Background}

We recall the notion of a classifying space for a family of subgroups (see e.g. Lück 2005). A family $\mathcal{F}$ of subgroups of a group $G$ is a non-empty set of subgroups that is closed under conjugation and finite intersections. The family consisting only of the trivial subgroup is denoted by $\mathcal{T} \mathcal{R}$. The family $\mathcal{F}\langle\mathcal{H}\rangle$ generated by a set of subgroups $\mathcal{H}$ is the smallest family containing $\mathcal{H}$. For a family $\mathcal{F}$ of subgroups of $G$ and a subgroup $H$ of $G$, we denote by $\left.\mathcal{F}\right|_{H}$ the family $\{L \cap H \mid L \in \mathcal{F}\}$ of subgroups of $H$. A classifying space $E_{\mathcal{F}} G$ for the family $\mathcal{F}$ is a terminal object in the $G$-homotopy category of $G$-CW-complexes with isotropy groups in $\mathcal{F}$. Note that a model for $E_{\mathcal{T} \mathcal{R}} G$ is given by $E G$. In particular, for every family $\mathcal{F}$ containing the trivial subgroup there exists a $G$-map $E G \rightarrow E_{\mathcal{F}} G$ that is unique up to $G$-homotopy.

Let $\mathcal{E} \subset \mathcal{F}$ be two families of subgroups of $G$. We say that $G$ satisfies condition $\left(M_{\mathcal{E} \subset \mathcal{F}}\right)$ if every element in $\mathcal{F} \backslash \mathcal{E}$ is contained in a unique maximal element $M$ in $\mathcal{F} \backslash \mathcal{E}$, and that $G$ satisfies condition $\left(N M_{\mathcal{E} \subset \mathcal{F}}\right)$ if additionally $M$ equals its normaliser $N_{G}(M)$. We denote by $W_{G}(M):=N_{G}(M) / M$ the Weyl group of $M$. The following proposition is a special case of a construction due to (Lück and Weiermann 2012, Corollary 2.8) stated in Li (2021, Corollary 2.2).

Theorem 2.1 (Lück-Weiermann) Let $G$ be a group and $\mathcal{E} \subset \mathcal{F}$ be two families of subgroups. Let $\left\{M_{i} \mid i \in I\right\}$ be a complete set of representatives for the conjugacy classes of maximal elements in $\mathcal{F} \backslash \mathcal{E}$.

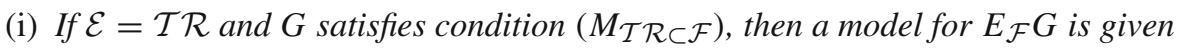
by the following G-pushout

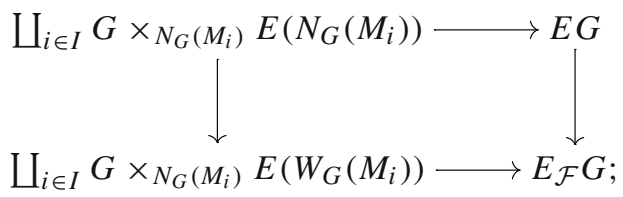

(ii) If $G$ satisfies conditions $\left(M_{\mathcal{E} \subset \mathcal{F}}\right)$ and $\left(N M_{\mathcal{E} \subset \mathcal{F}}\right)$, then a model for $E_{\mathcal{F}} G$ is given by the following G-pushout

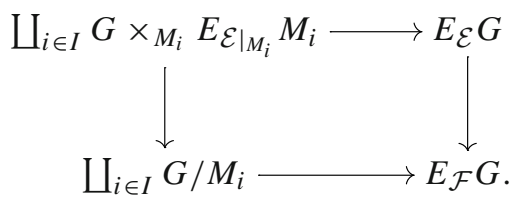


The $\mathcal{F}$-restricted orbit category $\mathcal{O}_{\mathcal{F}} G$ has $G$-sets $G / H$ with $H \in \mathcal{F}$ as objects and $G$-maps as morphisms. Let $A$ be an $\mathcal{O}_{\mathcal{F}} G$-module, that is a contravariant functor from the orbit category $\mathcal{O}_{\mathcal{F}} G$ to the category of modules. The equivariant cellular cohomology $H_{G}^{*}(X ; A)$ of a $G$-CW-complex $X$ with isotropy groups in $\mathcal{F}$ is called Bredon cohomology (Bredon 1967). In particular, Bredon cohomology satisfies the Mayer-Vietoris axiom for $G$-pushouts. Moreover, if $X$ is of the form $G \times{ }_{H} Y$ for a subgroup $H \subset G$ and an $H$-CW-complex $Y$, then there is a natural induction isomorphism (see e.g. Lück 2005)

$$
H_{G}^{*}\left(G \times{ }_{H} Y ; A\right) \cong H_{H}^{*}(Y ; A) .
$$

Here on the right hand side, $A$ is regarded as an $\left.\mathcal{O}_{\mathcal{F}}\right|_{H} H$-module by restriction along the obvious functor $\mathcal{O}_{\left.\mathcal{F}\right|_{H}} H \rightarrow \mathcal{O}_{\mathcal{F}} G$.

The (higher) topological complexity $\mathrm{TC}_{r}(\Gamma)$ of a group $\Gamma$ for $r \geq 2$ can be characterised in terms of classifying spaces for families (Farber and Oprea 2019, Theorem 3.1), generalising a result of Farber et al. (2019, Theorem 3.3) for the case $r=2$. Consider $G=\Gamma^{r}$ and let $\mathcal{D}$ be the family of subgroups of $\Gamma^{r}$ that is generated by the diagonal subgroup $\Delta(\Gamma)$ and the trivial subgroup.

Theorem 2.2 (Farber-Oprea) Let $\Gamma$ be a group and $r \geq 2$. Then $\mathrm{TC}_{r}(\Gamma)$ equals the infimum of integers $n$ for which the canonical $\Gamma^{r}$-map

$$
E\left(\Gamma^{r}\right) \rightarrow E_{\mathcal{D}}\left(\Gamma^{r}\right)
$$

is $\Gamma^{r}$-equivariantly homotopic to a $\Gamma^{r}$-map with values in the $n$-skeleton of $E_{\mathcal{D}}\left(\Gamma^{r}\right)$.

As a consequence (Farber and Oprea 2019, Theorem 5.1), a lower bound for $\mathrm{TC}_{r}(\Gamma)$ is given by the supremum of integers $n$ for which the canonical $\Gamma^{r}$-map $E\left(\Gamma^{r}\right) \rightarrow E_{\mathcal{D}}\left(\Gamma^{r}\right)$ induces a non-trivial map in Bredon cohomology

$$
H_{\Gamma^{r}}^{n}\left(E_{\mathcal{D}}\left(\Gamma^{r}\right) ; A\right) \rightarrow H_{\Gamma^{r}}^{n}\left(E\left(\Gamma^{r}\right) ; A\right)
$$

for some $\mathcal{O}_{\mathcal{D}}\left(\Gamma^{r}\right)$-module $A$.

\section{Proofs}

Fix an integer $r \geq 2$. Let $\Gamma$ be a group and $\Delta: \Gamma \rightarrow \Gamma^{r}$ be the diagonal map. For $\gamma=\left(\gamma_{1}, \ldots, \gamma_{r-1}\right) \in \Gamma^{r-1}$ and a subset $S \subset \Gamma$, we define the subgroup $H_{\gamma, S}$ of $\Gamma^{r}$ as

$$
H_{\gamma, S}:=\left(\gamma_{1}, \ldots, \gamma_{r-1}, e\right) \cdot \Delta\left(C_{\Gamma}(S)\right) \cdot\left(\gamma_{1}^{-1}, \ldots, \gamma_{r-1}^{-1}, e\right)
$$

Here $C_{\Gamma}(S)$ denotes the centraliser of $S$ in $\Gamma$. For $b \in \Gamma$, we write $H_{\gamma, b}$ instead of $H_{\gamma,\{b\}}$. Denote the element $\underline{e}:=(e, \ldots, e) \in \Gamma^{r-1}$ and note that $H_{\underline{e}, e}=\Delta(\Gamma)$. The elementary proof of the following lemma is omitted.

Lemma 3.1 Let $\gamma=\left(\gamma_{1}, \ldots, \gamma_{r-1}\right) \in \Gamma^{r-1}$ and $S \subset \Gamma$ be a subset. The following hold: 
(i) For $g=\left(g_{1}, \ldots, g_{r}\right) \in \Gamma^{r}$, let $\gamma^{\prime}:=\left(g_{1} \gamma_{1} g_{r}^{-1}, \ldots, g_{r-1} \gamma_{r-1} g_{r}^{-1}\right) \in \Gamma^{r-1}$ and $S^{\prime}:=\left\{g_{r} s g_{r}^{-1} \in \Gamma \mid s \in S\right\} \subset \Gamma$. Then we have

$$
g \cdot H_{\gamma, S} \cdot g^{-1}=H_{\gamma^{\prime}, S^{\prime}}
$$

(ii) For $\delta=\left(\delta_{1}, \ldots, \delta_{r-1}\right) \in \Gamma^{r-1}$ and a subset $T \subset \Gamma$, we have

$$
H_{\gamma, S} \cap H_{\delta, T}=H_{\gamma, S \cup T \cup\left\{\delta_{1}^{-1} \gamma_{1}, \ldots, \delta_{r-1}^{-1} \gamma_{r-1}\right\}} ;
$$

(iii) We have

$$
\begin{aligned}
N_{\Gamma^{r}}\left(H_{\gamma, S}\right)= & \left\{\left(\gamma_{1} k_{1} h \gamma_{1}^{-1}, \ldots, \gamma_{r-1} k_{r-1} h \gamma_{r-1}^{-1}, h\right) \in \Gamma^{r} \mid h \in N_{\Gamma}\left(C_{\Gamma}(S)\right),\right. \\
& \left.k_{1}, \ldots, k_{r-1} \in C_{\Gamma}\left(C_{\Gamma}(S)\right)\right\} .
\end{aligned}
$$

Let $\mathcal{F}_{1} \subset \mathcal{D}$ be the families of subgroups of $\Gamma^{r}$ defined as

$$
\begin{aligned}
\mathcal{D} & :=\mathcal{F}\langle\{\Delta(\Gamma)\}\rangle \cup \mathcal{T} \mathcal{R} ; \\
\mathcal{F}_{1} & :=\mathcal{F}\left\langle\left\{H_{\gamma, b} \mid \gamma \in \Gamma^{r-1}, b \in \Gamma \backslash\{e\}\right\}\right\rangle \cup \mathcal{T} \mathcal{R} .
\end{aligned}
$$

From now on, let $\Gamma$ be a group as in Theorem 1.1. The following properties of centralisers in $\Gamma$ will be used in the sequel. For $b, c \in \Gamma \backslash\{e\}$, we have either $C_{\Gamma}(b)=C_{\Gamma}(c)$ or $C_{\Gamma}(b) \cap C_{\Gamma}(c)=\{e\}$ by (Li 2021, Lemma 3.4). By assumption on $\Gamma$, the centraliser $C_{\Gamma}(b)$ of an element $b \in \Gamma \backslash\{e\}$ is infinite cyclic or isomorphic to one of the $P_{i}$. In both cases we have $N_{\Gamma}\left(C_{\Gamma}(b)\right)=C_{\Gamma}\left(C_{\Gamma}(b)\right)=C_{\Gamma}(b)$ (see Li 2021, Lemma 3.5) for more details.

Lemma 3.2 Let $\Gamma$ be a group as in Theorem 1.1 and let $\underline{e}=(e, \ldots, e) \in \Gamma^{r-1}$. Then for $n=\operatorname{cd}\left(\Gamma^{r}\right)$ and every $\mathcal{O}_{\mathcal{D}}\left(\Gamma^{r}\right)$-module $A$, we have

$$
H_{\Gamma^{r}}^{n}\left(\Gamma^{r} \times{ }_{H_{\underline{e}, e}} E_{\left.\mathcal{F}_{1}\right|_{H_{\underline{e}, e}}}\left(H_{\underline{e}, e}\right) ; A\right)=0 .
$$

Proof We have that conditions $\left(M_{\left.\mathcal{T} \mathcal{R} \subset \mathcal{F}_{1}\right|_{H_{e}, e}}\right)$ and $\left(N M_{\left.\mathcal{T} \mathcal{R} \subset \mathcal{F}_{1}\right|_{H_{\underline{e}, e}}}\right)$ hold for the group $H_{\underline{e}, e}$. To see this, we note that under the obvious isomorphism $H_{\underline{e}, e} \cong \Gamma$, the family $\left.\mathcal{F}_{1}\right|_{H_{e}, e}$ is identified with $\mathcal{F}\left\langle\left\{C_{\Gamma}(b) \mid b \in \Gamma \backslash\{e\}\right\}\right\rangle \cup \mathcal{T} \mathcal{R}$, using Lemma 3.1(i) and (ii). By Theorem 2.1(ii), we obtain an $H_{\underline{e}, e}$-pushout

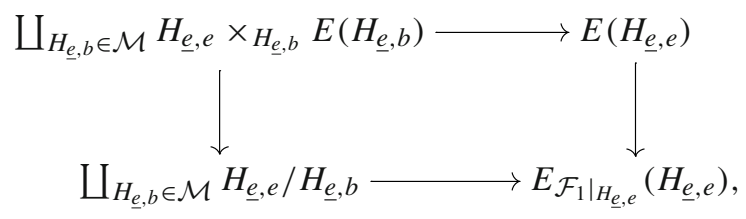

where $\mathcal{M}$ is a complete set of representatives of conjugacy classes of maximal elements in $\left.\mathcal{F}_{1}\right|_{H_{e}, e} \backslash \mathcal{T} \mathcal{R}$. Since $\operatorname{cd}\left(H_{\underline{e}, e}\right)<n$ and $\operatorname{cd}\left(H_{\underline{e}, b}\right)<n-1$ for $b \in \Gamma \backslash\{e\}$, the Mayer-Vietoris sequence for $H_{H_{e, e}}^{*}(-; A)$ applied to the pushout (2) together with the induction isomorphism (1) yields the lemma. 
Proof of Theorem 1.1 Throughout the proof let $n=\operatorname{cd}\left(\Gamma^{r}\right)$. We show that the $\Gamma^{r}$-map $E\left(\Gamma^{r}\right) \rightarrow E_{\mathcal{D}}\left(\Gamma^{r}\right)$ induces a surjective map $H_{\Gamma^{r}}^{n}\left(E_{\mathcal{D}}\left(\Gamma^{r}\right) ; A\right) \rightarrow H_{\Gamma^{r}}^{n}\left(E\left(\Gamma^{r}\right) ; A\right)$ for every $\mathcal{O}_{\mathcal{D}}\left(\Gamma^{r}\right)$-module $A$. Then the theorem follows from Theorem 2.2 and the upper bound $\mathrm{TC}_{r}(\Gamma) \leq n$ (see e.g. Farber and Oprea 2019, (4)).

First, observe that condition $\left(M_{\mathcal{T} \mathcal{R} \subset \mathcal{F}_{1}}\right)$ holds, since conjugates of the subgroups generating $\mathcal{F}_{1}$ either coincide or have trivial intersection by Lemma 3.1(i) and (ii). Moreover, for $\gamma \in \Gamma^{r-1}$ and $b \in \Gamma \backslash\{e\}$ there is an isomorphism $N_{\Gamma^{r}}\left(H_{\gamma, b}\right) \cong C_{\Gamma}(b)^{r}$ by Lemma 3.1(iii). In particular, we have $\operatorname{cd}\left(N_{\Gamma^{r}}\left(H_{\gamma, b}\right)\right)<n$, since

$$
n \geq \operatorname{cd}\left(\Gamma \times \mathbb{Z}^{r-1}\right)=\operatorname{cd}(\Gamma)+r-1>r
$$

and $n>\operatorname{cd}\left(P_{i}^{r}\right)$ by assumption. Then it follows from the induction isomorphism (1) that we have

$$
H_{\Gamma^{r}}^{n}\left(\Gamma^{r} \times_{N_{\Gamma^{r}}\left(H_{\gamma, b}\right)} E\left(N_{\Gamma^{r}}\left(H_{\gamma, b}\right)\right) ; A\right)=0
$$

for every $\mathcal{O}_{\mathcal{D}}\left(\Gamma^{r}\right)$-module $A$. By Theorem 2.1(i), we obtain a $\Gamma^{r}$-pushout

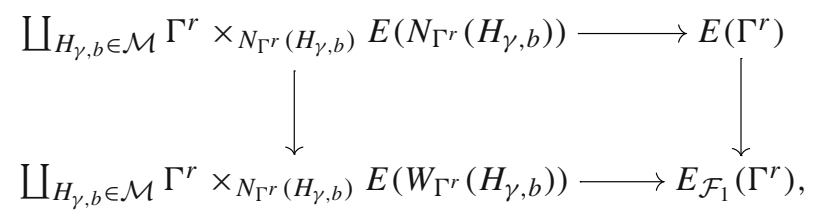

where $\mathcal{M}$ is a complete set of representatives of conjugacy classes of maximal elements in $\mathcal{F}_{1} \backslash \mathcal{T} \mathcal{R}$. Applying the Mayer-Vietoris sequence for $H_{\Gamma^{r}}^{*}(-; A)$ to the pushout (3) shows that the induced map $H_{\Gamma^{r}}^{n}\left(E_{\mathcal{F}_{1}}\left(\Gamma^{r}\right) ; A\right) \rightarrow H_{\Gamma^{r}}^{n}\left(E\left(\Gamma^{r}\right) ; A\right)$ is surjective.

Second, observe that condition $\left(M_{\mathcal{F}_{1} \subset \mathcal{D}}\right)$ holds, since by Lemma 3.1(i) and (ii), two conjugates of $\Delta(\Gamma)$ in $\Gamma^{r}$ either coincide or their intersection lies in $\mathcal{F}_{1}$. Moreover, condition $\left(N M_{\mathcal{F}_{1} \subset \mathcal{D}}\right)$ holds by Lemma 3.1(iii), using that the centre $C_{\Gamma}(\Gamma)$ of $\Gamma$ is trivial. By Theorem 2.1(ii), we obtain a $\Gamma^{r}$-pushout

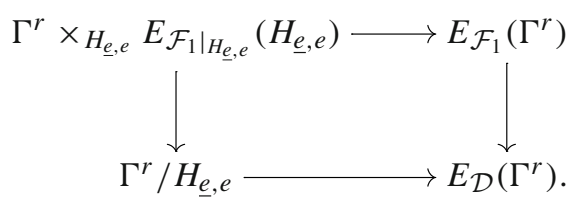

Then Lemma 3.2 and the Mayer-Vietoris sequence for $H_{\Gamma^{r}}^{*}(-; A)$ applied to the pushout (4) yield that the map $H_{\Gamma^{r}}^{n}\left(E_{\mathcal{D}}\left(\Gamma^{r}\right) ; A\right) \rightarrow H_{\Gamma^{r}}^{n}\left(E_{\mathcal{F}_{1}}\left(\Gamma^{r}\right) ; A\right)$ is surjective.

Together, the map $H_{\Gamma^{r}}^{n}\left(E_{\mathcal{D}}\left(\Gamma^{r}\right) ; A\right) \rightarrow H_{\Gamma^{r}}^{n}\left(E\left(\Gamma^{r}\right) ; A\right)$ is surjective for every $\mathcal{O}_{\mathcal{D}}\left(\Gamma^{r}\right)$-module $A$. This finishes the proof.

Proof of Corollary 1.2 For groups $\Gamma$ of type $F$, we have $\operatorname{cd}\left(\Gamma^{r}\right)=r \cdot \operatorname{cd}(\Gamma)$ by Dranishnikov (2019, Corollary 2.5). The result now follows from Theorem 1.1. 
Proof of Corollary 1.3 The result follows from Corollary 1.2 using the fact that torsionfree hyperbolic groups are of type $F$ (see e.g. Bridson and Haefliger 1999, Corollary 3.26 on p. 470).

Acknowledgements Both authors would like to thank the organisers of the workshop "Advances in Homotopy Theory" held in September 2021 at the Southampton Centre for Geometry, Topology, and Applications which inspired this work. The first author was supported by the Engineering and Physical Sciences Research Council (Grant Number 2127970) and the European Research Council under the European Union's Horizon 2020 research and innovation programme (Grant Number 850930). We thank the referees for helpful comments.

Open Access This article is licensed under a Creative Commons Attribution 4.0 International License, which permits use, sharing, adaptation, distribution and reproduction in any medium or format, as long as you give appropriate credit to the original author(s) and the source, provide a link to the Creative Commons licence, and indicate if changes were made. The images or other third party material in this article are included in the article's Creative Commons licence, unless indicated otherwise in a credit line to the material. If material is not included in the article's Creative Commons licence and your intended use is not permitted by statutory regulation or exceeds the permitted use, you will need to obtain permission directly from the copyright holder. To view a copy of this licence, visit http://creativecommons.org/licenses/by/4.0/.

\section{References}

Aguilar-Guzmán, J., González, J., Oprea, J.: Right-angled Artin groups, polyhedral products and the TCgenerating function. Proc. R. Soc. Edinb. A (2021). https://doi.org/10.1017/prm.2021.26

Bredon, G.E.: Equivariant Cohomology Theories. Lecture Notes in Mathematics, No. 34. Springer, BerlinNew York (1967)

Bridson, M.R., Haefliger, A.: Metric Spaces of Non-positive Curvature. Grundlehren der Mathematischen Wissenschaften, vol. 319. Springer, Berlin (1999)

Dranishnikov, A.: On dimension of product of groups. Algebra Discrete Math. 28(2), 203-212 (2019)

Dranishnikov, A.: On topological complexity of hyperbolic groups. Proc. Am. Math. Soc. 148(10), 4547$4556(2020)$

Farber, M.: Topological complexity of motion planning. Discrete Comput. Geom. 29(2), 211-221 (2003)

Farber, M., Mescher, S.: On the topological complexity of aspherical spaces. J. Topol. Anal. 12(2), 293-319 (2020)

Farber, M., Oprea, J.: Higher topological complexity of aspherical spaces. Topology Appl. 258, 142-160 (2019)

Farber, M., Grant, M., Lupton, G., Oprea, J.: Bredon cohomology and robot motion planning. Algebr. Geom. Topol. 19(4), 2023-2059 (2019)

Farber, M., Kishimoto, D., Stanley, D.: Generating functions and topological complexity. Topology Appl. 278, 107235, 5 (2020)

González, J., Gutiérrez, B., Yuzvinsky, S.: Higher topological complexity of subcomplexes of products of spheres and related polyhedral product spaces. Topol. Methods Nonlinear Anal. 48(2), 419-451 (2016)

Li, K.: On the topological complexity of toral relatively hyperbolic groups. Proc. Am. Math. Soc. (2021). https://doi.org/10.1090/proc/15733

Lück, W.: Survey on classifying spaces for families of subgroups. In: Infinite Groups: Geometric, Combinatorial and Dynamical Aspects, vol. 248. Progr. Math., pp. 269-322. Birkhäuser, Basel (2005)

Lück, W.: Equivariant cohomological Chern characters. Int. J. Algebra Comput. 15(5-6), 1025-1052 (2005)

Lück, W., Weiermann, M.: On the classifying space of the family of virtually cyclic subgroups. Pure Appl. Math. Q. 8(2), 497-555 (2012)

Rudyak, Y.B.: On higher analogs of topological complexity. Topol. Appl. 157(5), 916-920 (2010)

Publisher's Note Springer Nature remains neutral with regard to jurisdictional claims in published maps and institutional affiliations. 